\title{
Late Gadolinium Enhancement imaging using stack of stars and compressed sensing
}

\author{
Ganesh Adluru, Liyong Chen, Seong-Eun Kim, Norman Hu, Kyle H Sabey, David A Bull, Eugene Kholmovski, \\ Nassir Marrouche, Edward DiBella*
}

From 2011 SCMR/Euro CMR Joint Scientific Sessions

Nice, France. 3-6 February 2011

\section{Objective}

To develop and test a 3D hybrid radial(stack of stars) acquisition scheme with compressed sensing(CS) reconstruction for high resolution late gadolinium enhancement(LGE) imaging of the left ventricle $(\mathrm{LV})$ and the left atrium(LA).

\section{Background}

LGE imaging is the gold standard for identifying viable tissue and fibrosis in the LV [1]. It has also been recently applied to imaging of the LA to identify regions of RF ablation [2] and fibrosis [3]. While a Cartesian acquisition scheme is commonly used, it can take a long time [1] and can be sensitive to motion leading to inconsistent image quality $[3,4]$. A radial acquisition scheme can be advantageous due to its robustness to motion and undersampling. Here a hybrid radial acquisition is combined with CS to improve LGE imaging efficiency.

\section{Methods}

A kz-first segmented acquisition was implemented on a Siemens 3T scanner. This scheme results in having consistent in-plane data with inconsistencies along kz leading to improved image quality as shown with simulation studies in [5]. As well, each plane is rotated by an angle based on golden ratio - Figure 1. This rotation helps exploit sparsity also along the z-dimension - a CS reconstruction with a 3D total variation constraint was used. Acquired data was first interpolated onto a 3D Cartesian grid and minimization of the cost function for CS was done with a gradient descent scheme.

The methods were tested for imaging the LV of three rabbits and for imaging the LA of three patients at least

University of Utah, Salt Lake City, UT, USA three months post-ablation. The scan parameters for the rabbit studies were $\mathrm{TR}=2.4 \mathrm{~ms}, \mathrm{TE}=2.7 \mathrm{~ms}$, voxel size $=0.9 \times 0.9 \times 2 \mathrm{~mm}^{3}, \mathrm{TI}=300-350 \mathrm{~ms}$. For the patient studies the scan parameters were $\mathrm{TR}=3.4 \mathrm{~ms}, \mathrm{TE}=1.83 \mathrm{~ms}$, voxel size $=1.25 \times 1.25 \times 2.5 \mathrm{~mm}^{3}, \mathrm{TI}=300-400 \mathrm{~ms} ; 36$ slices with 144 rays in each plane were acquired in $1.25-2 \mathrm{~min}$.

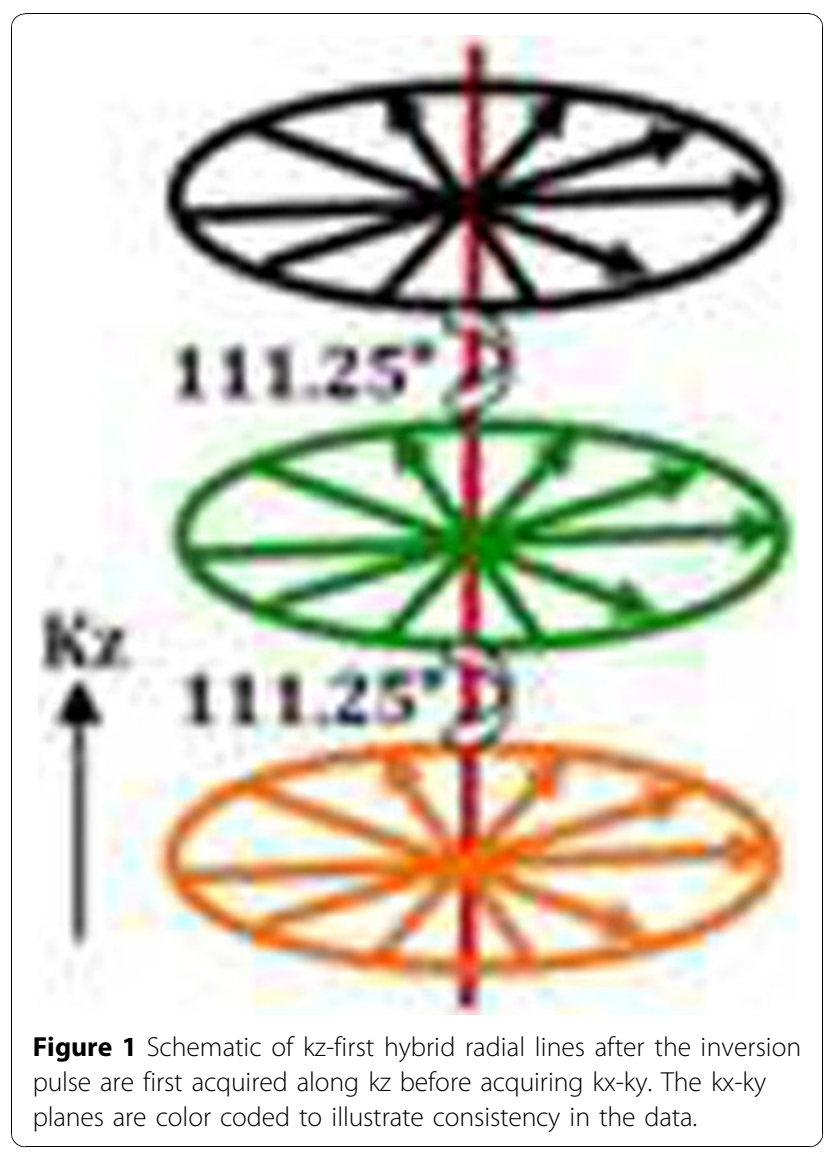




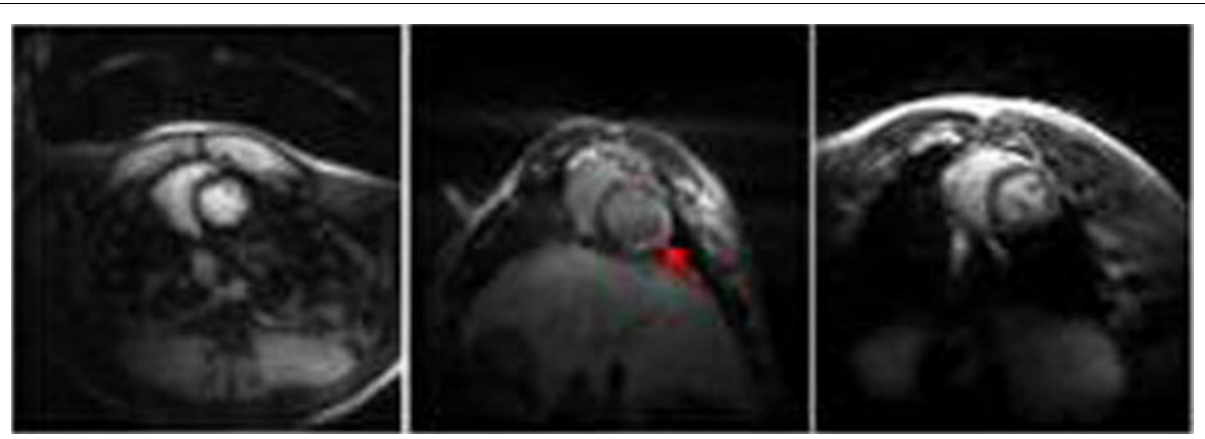

Figure 2 Results If LGE imaging of the LV with proposed scheme. One representative slice from each of the three studies is shown. Arrow in the image points to enhancement.
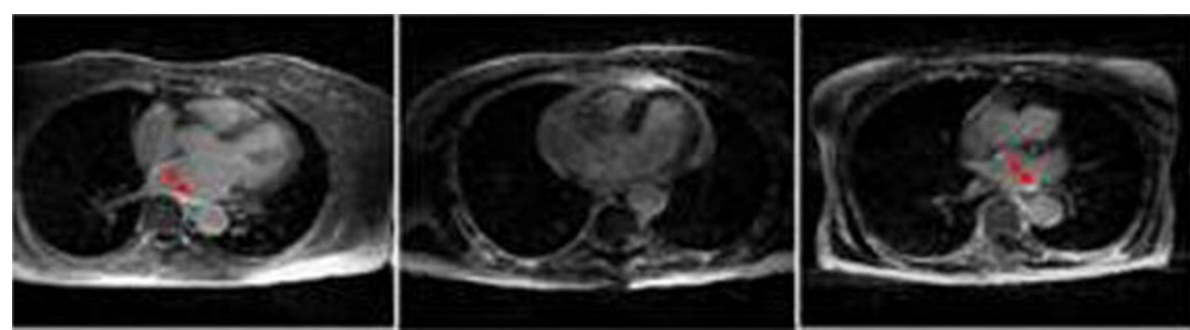

Figure 3 Results of LGE imaging of the LA. One representative slice from each of the three studies is shown. Arrows in the images point to enhancement.

\section{Results}

Figure 2 shows the results from three rabbit studies. Enhancement in the lateral free wall corresponds to tissue necrosis from a ligation of a coronary artery. Axial slices of LA from three patient studies are shown in Figure 3. Enhancement corresponds to ablated regions in the atrium.

\section{Conclusion}

The 3D stack of stars acquisition in conjunction with CS offers a promising alternative for rapid high resolution LGE imaging. More patient studies are required to validate the proposed framework.

\section{Published: 2 February 2011}

\section{References}

1. Simonetti OP, et al: Radiol 2001, 218:215-223.

2. Peters DC, et al: Radiol 2007, 243:690-695

3. Oakes RS, et al: Circulation 2009, 119:1758-1767.

4. Peters DC, et al: JACC Cardiovasc Imaging 2009, 2:308-316.

5. Adluru G, et al: ISMRM 2010, 1285.

doi:10.1186/1532-429X-13-S1-P162

Cite this article as: Adluru et al: Late Gadolinium Enhancement imaging using stack of stars and compressed sensing. Journal of Cardiovascular Magnetic Resonance 2011 13(Suppl 1):P162.

\section{Submit your next manuscript to BioMed Central} and take full advantage of:

- Convenient online submission

- Thorough peer review

- No space constraints or color figure charges

- Immediate publication on acceptance

- Inclusion in PubMed, CAS, Scopus and Google Scholar

- Research which is freely available for redistribution
Biomed Central 